\title{
CONVERGENCE OF AN ENERGY-PRESERVING SCHEME FOR THE ZAKHAROV EQUATIONS IN ONE SPACE DIMENSION
}

\author{
R. T. GLASSEY
}

\begin{abstract}
An energy-preserving, linearly implicit finite difference scheme is presented for approximating solutions to the periodic Cauchy problem for the one-dimensional Zakharov system of two nonlinear partial differential equations. First-order convergence estimates are obtained in a standard "energy" norm in terms of the initial errors and the usual discretization errors.
\end{abstract}

\section{INTRODUCTION}

In [11] Zakharov introduced a system of equations to model the propagation of Langmuir waves in a plasma. If we denote by $N(x, t) \quad(x \in \mathbb{R}, t>0)$ the deviation of the ion density from its equilibrium value, and by $E(x, t)$ the envelope of the high-frequency electric field, then the one-dimensional system takes the form

$$
i E_{t}+E_{x x}=N E
$$

$$
N_{t t}-N_{x x}=\frac{\partial^{2}}{\partial x^{2}}\left(|E|^{2}\right) .
$$

We solve on $\{x \in \mathbb{R}, t>0\}$ and supplement (ZS) by prescribing initial values for $E, N$, and $N_{t}$ :

$$
E(x, 0)=E^{0}(x), \quad N(x, 0)=N^{0}(x), \quad N_{t}(x, 0)=N^{1}(x) .
$$

Most of the interest to date in (ZS) stems from two particular features. Firstly, (ZS) admits solitary wave solutions [3]. Secondly, in three space dimensions, (ZS) was derived to model the collapse of caverns (cf. [11]). An intriguing and still unresolved question remains in three dimensions as to whether smooth data can generate a solution which becomes singular in finite time.

As is well known, (ZS) possesses the two formal invariants

$$
\begin{gathered}
\int_{-\infty}^{\infty}|E(x, t)|^{2} d x=\int_{-\infty}^{\infty}|E(x, 0)|^{2} d x, \\
\int_{-\infty}^{\infty}\left(\left|E_{x}\right|^{2}+\frac{1}{2}\left(|v|^{2}+N^{2}\right)+N|E|^{2}\right) d x=\text { const },
\end{gathered}
$$

Received July 17, 1990; revised November 20, 1990.

1991 Mathematics Subject Classification. Primary 65M12, 35L70.

Research supported in part by NSF grant DMS 8721721. 
where $v$ is given by

$$
v=-u_{x}, \quad u_{x x}=N_{t} .
$$

We know that these are sufficient for global weak existence (cf. [9]). Also from [9] the same conclusion holds in three dimensions under an additional "smallness" condition. Moreover, higher-order estimates from [9] guarantee the existence of a smooth solution in one dimension provided smooth data are prescribed.

It is such a smooth solution of (ZS) with periodic boundary conditions which we approximate numerically in this paper. A spectral method is used in [5]; while practical results seem very good, the convergence issue is not rigorously addressed. Our algorithm uses an approximation of "Crank-Nicolson" type on the linear parts of (ZS). We approximate the solution over a fixed but arbitrary time interval $0 \leq t \leq T$.

The nonlinear terms in (ZS) are then approximated in such a way that:

(i) the discrete $L^{2}$-norm (over a period) of the approximation to $E$ is conserved; and

(ii) a discrete analogue of the total energy is conserved.

This discrete energy will be shown to be bounded below by a positive definite form. The scheme is linearly implicit and involves only two periodic tridiagonal solvers to advance one step in time. We obtain first-order convergence estimates in the natural "energy norm" in terms of initial errors and standard discretization errors.

In the references we list several papers where conservative schemes have been employed [2, 4, 6, 8]. Related results are to be found in [1, 10].

The standard summation by parts formula is

$$
\begin{aligned}
\sum_{j=1}^{J} v_{j}\left(u_{j+1}-2 u_{j}+u_{j-1}\right)= & v_{J+1}\left(u_{J+1}-u_{J}\right)-v_{1}\left(u_{1}-u_{0}\right) \\
& -\sum_{j=1}^{J}\left(v_{j+1}-v_{j}\right)\left(u_{j+1}-u_{j}\right) .
\end{aligned}
$$

The "summed" terms cancel whenever $\left\{u_{k}\right\},\left\{v_{k}\right\}$ are $J$-periodic mesh functions.

Although [9] treats the Cauchy problem on all of space, the methods given there (i.e., Galerkin) could be extended to deal with the periodic case studied here. Constants depending on $T$ and the Cauchy data are written $c_{T}$, while constants depending only on the data are generically written as $c$. These will change from line to line without explicit mention.

This scheme has been implemented; details will appear elsewhere.

\section{THE FINITE DIFFERENCE SCHEME}

Let $T>0$ be arbitrary; we will approximate the solution to the periodic Cauchy problem for (ZS) over the time interval $0 \leq t \leq T$. We first state hypotheses on the Cauchy data and the solution:

(H0) The Cauchy data

$$
E(x, 0)=E^{0}(x), \quad N(x, 0)=N^{0}(x), \quad N_{t}(x, 0)=N^{1}(x)
$$


are $C^{\infty}$ and $L$-periodic. Moreover,

$$
\begin{gathered}
\int_{0}^{L} N^{1}(x) d x=0, \\
\sum_{j=1}^{J} N^{1}(j h)=0 \text { for any } h>0 \text { with } J h=L .
\end{gathered}
$$

(HE) The periodic Cauchy problem possesses a unique smooth global solution.

In order to write the scheme, we define

$$
\begin{gathered}
\delta u_{k} \equiv \Delta x^{-1}\left(u_{k+1}-u_{k}\right), \\
\delta^{2} u_{k} \equiv \Delta x^{-2}\left(u_{k+1}-2 u_{k}+u_{k-1}\right), \\
\lambda=\frac{\Delta t}{\Delta x}, \quad \beta=\frac{\Delta t}{\Delta x^{2}}
\end{gathered}
$$

with $\Delta t, \Delta x>0$. Now for $J$ a positive integer we choose $\Delta x=\frac{L}{J}, \Delta t>0$ such that

$$
n \Delta t \leq T
$$

and define $t^{l}=l \Delta t, x_{j}=j \Delta x \quad(l=0, \ldots, n ; j=0, \ldots, J)$.

Our scheme is

$$
\begin{gathered}
i \frac{E_{k}^{n+1}-E_{k}^{n}}{\Delta t}+\frac{1}{2} \delta^{2} E_{k}^{n}+\frac{1}{2} \delta^{2} E_{k}^{n+1}=\frac{1}{4}\left(N_{k}^{n}+N_{k}^{n+1}\right)\left(E_{k}^{n}+E_{k}^{n+1}\right), \\
\frac{N_{k}^{n+1}-2 N_{k}^{n}+N_{k}^{n-1}}{\Delta t^{2}}-\frac{1}{2} \delta^{2} N_{k}^{n+1}-\frac{1}{2} \delta^{2} N_{k}^{n-1}=\delta^{2}\left(\left|E_{k}^{n}\right|^{2}\right) .
\end{gathered}
$$

In both relations $k=1, \ldots, J, n \geq 0$ in the first and $n \geq 1$ in the second. Here we take $E_{k}^{n}, N_{k}^{n}$ to be $J$-periodic mesh functions, i.e.,

$$
E_{k}^{n}=E_{j}^{n}, \quad N_{k}^{n}=N_{j}^{n} \quad \text { if } k \equiv j(\bmod J) .
$$

The scheme is supplemented with the initial values

$$
N_{k}^{0}=N^{0}\left(x_{k}\right), \quad N_{k}^{1}=N_{k}^{0}+\Delta t N^{1}\left(x_{k}\right) .
$$

We claim that the scheme is uniquely solvable: multiplying $(8 . N)$ by $\Delta t^{2}$, we see that the coefficient matrix for the unknown $\left\{N_{k}^{n+1}\right\}_{k=1}^{J}$, of order $J \times J$, is

$$
A_{N}=\left[\begin{array}{ccccc}
1+\lambda^{2} & -\frac{\lambda^{2}}{2} & 0 & \cdots & -\frac{\lambda^{2}}{2} \\
-\frac{\lambda^{2}}{2} & 1+\lambda^{2} & -\frac{\lambda^{2}}{2} & \cdots & 0 \\
\vdots & \vdots & \vdots & \ddots & \vdots \\
-\frac{\lambda^{2}}{2} & 0 & \cdots & -\frac{\lambda^{2}}{2} & 1+\lambda^{2}
\end{array}\right] \text {, }
$$

which is invertible by Gerschgorin for any $\lambda>0$. The coefficient matrix for the unknown $\left\{E_{k}^{n+1}\right\}_{k=1}^{J}$ has the form

$$
\text { iI }-A_{E} \text {, }
$$

where both matrices are square and of order $J \times J$. 
$A_{E}$ is symmetric and has the form

$$
A_{E}=\left(\begin{array}{ccccc}
\left(A_{E}\right)_{11} & -\frac{\beta}{2} & 0 & \ldots & -\frac{\beta}{2} \\
-\frac{\beta}{2} & \left(A_{E}\right)_{22} & -\frac{\beta}{2} & \ldots & 0 \\
\vdots & \vdots & \ddots & \vdots & \vdots \\
-\frac{\beta}{2} & 0 & \ldots & -\frac{\beta}{2} & \left(A_{E}\right)_{J J}
\end{array}\right),
$$

where

$$
\left(A_{E}\right)_{k k}=\beta+\frac{\Delta t}{4}\left(N_{k}^{n}+N_{k}^{n+1}\right) .
$$

Since $A_{E}$ has only real eigenvalues, $i I-A_{E}$ is invertible. Thus the scheme is uniquely solvable at each time step. Indeed, putting $n=0$ in (8.E), we can solve for $\left\{E_{k}^{1}\right\}$, since $N_{k}^{0}, N_{k}^{1}, E_{k}^{0}$ are known from the data. Putting $n=1$ in (8.N), we can then solve for $\left\{N_{k}^{2}\right\}$ and, using $\left\{N_{k}^{2}\right\}$, we can put $n=1$ in (8.E) and solve for $\left\{E_{k}^{2}\right\}$, etc.

We summarize with

Lemma 1. Assume the data satisfy (H0). Then the scheme (8.E), (8.N) is uniquely solvable at each time step.

Lemma 2. Let the data satisfy (H0). Define $\left\{u_{k}^{n}\right\}$ by

$$
\frac{u_{k+1}^{n}-2 u_{k}^{n}+u_{k-1}^{n}}{\Delta x^{2}}=\frac{N_{k}^{n+1}-N_{k}^{n}}{\Delta t}, \quad k=1, \ldots, J-1,
$$

Extend $\left\{u_{k}^{n}\right\}$ by defining

$$
u_{k}^{n}=u_{j}^{n} \quad \text { if } \quad k \equiv j(\bmod J) .
$$

Then

$$
u_{k}^{n}=-\Delta x \sum_{j=1}^{J-1} G\left(x_{k}, x_{j}\right) \frac{N_{j}^{n+1}-N_{j}^{n}}{\Delta t},
$$

where

$$
G(x, y)= \begin{cases}x\left(1-\frac{y}{L}\right), & 0 \leq x \leq y \leq L, \\ y\left(1-\frac{x}{L}\right), & 0 \leq y \leq x \leq L .\end{cases}
$$

Proof. The proof that the given representation is indeed a solution is a straightforward computation and is omitted. The only issue is one of compatibility. Summing the definition of $u_{k}^{n}$, we see that it is required that

$$
\sum_{k=1}^{J}\left(N_{k}^{n+1}-N_{k}^{n}\right)=0
$$

When $n=0$, this is true by hypotheses (H0) and (10). Using $(8 . N)$, we can write

$$
N_{k}^{n+1}-N_{k}^{n}=N_{k}^{n}-N_{k}^{n-1}+\frac{\Delta t^{2}}{2} \delta^{2}\left(N_{k}^{n+1}+N_{k}^{n-1}+2\left|E_{k}^{n}\right|^{2}\right) .
$$

Using induction, we sum both sides over $k$. The sum of the first two terms on the right vanishes by the induction hypothesis; the sum of the remaining terms vanishes by periodicity. 
Theorem 1. Let the data satisfy (H0). Then the scheme (8) possesses the following two invariants:

(a)

$$
\sum_{k}\left|E_{k}^{n}\right|^{2} \Delta x=\text { const } \quad(n \Delta t \leq T) .
$$

(b) Define $u_{k}^{n}$ as in Lemma 2, so that $\delta^{2} u_{k}^{n}=\left(N_{k}^{n+1}-N_{k}^{n}\right) / \Delta t$. Then

$$
\begin{aligned}
\mathscr{E}_{d}^{n+1} \equiv \Delta x \sum_{k}\left[\left|\delta E_{k}^{n+1}\right|^{2}+\frac{1}{2}\left(\delta u_{k}^{n}\right)^{2}+\frac{1}{4}\left\{\left(N_{k}^{n}\right)^{2}\right.\right. & \left.+\left(N_{k}^{n+1}\right)^{2}\right\} \\
& \left.+\frac{1}{2}\left(N_{k}^{n}+N_{k}^{n+1}\right)\left|E_{k}^{n+1}\right|^{2}\right]=\text { const }
\end{aligned}
$$

for $n \Delta t \leq T$. The sums run over $1 \leq k \leq J$.

Thus the discrete $L^{2}$-norm of $E^{n}$ over a period is conserved, and the form of $\mathscr{E}_{d}^{n}$ is similar to that for the exact solution in (2), (3).

We show that $\mathscr{E}_{d}^{n}$ is bounded below by a positive definite form. For this purpose, we put

$$
\begin{aligned}
\left\|E^{n}\right\|_{2}^{2} & \equiv \sum_{k}\left|E_{k}^{n}\right|^{2} \Delta x, \\
\left\|\delta E^{n}\right\|_{2}^{2} & \equiv \sum_{k}\left|\delta E_{k}^{n}\right|^{2} \Delta x,
\end{aligned}
$$

with similar quantities for $N^{n}$. We make note of the discrete Sobolev inequality

$$
\sup _{k}\left|u_{k}\right| \leq c\|u\|_{2}^{1 / 2}\|\delta u\|_{2}^{1 / 2}
$$

valid for periodic mesh functions $\left\{u_{k}\right\}$. Indeed, denoting the Fourier coefficients of the mesh function $u$ by $\left\{c_{m}\right\}$, we write

$$
\begin{aligned}
\left|u_{k}\right| & \leq c\left(\sum_{|m| \leq M}+\sum_{|m|>M}\right)\left|c_{m}\right| \\
& \leq c M^{1 / 2}\left(\sum_{m}\left|c_{m}\right|^{2}\right)^{1 / 2}+c M^{-(1 / 2)}\left(\sum_{m}|m|^{2}\left|c_{m}\right|^{2}\right)^{1 / 2}
\end{aligned}
$$

and optimize on $M$.

The last term $\mathscr{L}$ in $\mathscr{E}_{d}^{n}$ is estimable by

$$
\begin{aligned}
|\mathscr{L}| & \leq \frac{1}{2} \sum_{k}\left|N_{k}^{n}\right|\left|E_{k}^{n+1}\right|^{2} \Delta x+\frac{1}{2} \sum_{k}\left|N_{k}^{n+1}\right|\left|E_{k}^{n+1}\right|^{2} \Delta x \\
& \leq \frac{\varepsilon}{4} \sum_{k}\left(\left(N_{k}^{n}\right)^{2}+\left(N_{k}^{n+1}\right)^{2}\right) \Delta x+\frac{1}{2 \varepsilon} \sum_{k}\left|E_{k}^{n+1}\right|^{4} \Delta x
\end{aligned}
$$

for any $\varepsilon>0$. Choosing $\varepsilon=\frac{1}{2}$, we get the bound

$$
|\mathscr{L}| \leq \frac{1}{8} \sum_{k} \Delta x\left(\left(N_{k}^{n}\right)^{2}+\left(N_{k}^{n+1}\right)^{2}\right)+\left\|E^{n+1}\right\|_{4}^{4} .
$$


By the Sobolev inequality (17) and part (a) of the theorem,

$$
\begin{aligned}
\left\|E^{n+1}\right\|_{4}^{4} & \leq c\left\|E^{n+1}\right\|_{2}^{2}\left\|E^{n+1}\right\|_{\infty}^{2} \leq c\left\|E^{n+1}\right\|_{\infty}^{2} \leq c\left\|\delta E^{n+1}\right\|_{2} \\
& \leq \frac{1}{4}\left\|\delta E^{n+1}\right\|_{2}^{2}+c .
\end{aligned}
$$

This gives us

Lemma 3. There is a constant $c$, depending only on the data, such that the solution of the discrete scheme (8.E), (8.N) satisfies

$$
\sum_{k} \Delta x\left[\left|E_{k}^{n+1}\right|^{2}+\left|\delta E_{k}^{n+1}\right|^{2}+\left(\delta u_{k}^{n}\right)^{2}+\left(N_{k}^{n}\right)^{2}+\left(N_{k}^{n+1}\right)^{2}\right] \leq c,
$$

and hence $\sup _{k}\left|E_{k}^{n}\right| \leq c$.

Proof of Theorem 1. As is well known, part (a) is obtained by multiplying (8.E) by $\bar{E}_{k}^{n+1}+\bar{E}_{k}^{n}$, summing over $k, k=1, \ldots, J$, and taking the imaginary part.

In order to verify (b), we multiply $(8 . E)$ by $\bar{E}_{k}^{n+1}-\bar{E}_{k}^{n}$ and sum on $k$. Adding this to its conjugate, we obtain

$$
I_{n}+I_{n+1}=\frac{1}{4} \sum_{k}\left(N_{k}^{n+1}+N_{k}^{n}\right) \cdot 2 \operatorname{Re}\left(E_{k}^{n+1}+E_{k}^{n}\right)\left(\bar{E}_{k}^{n+1}-\bar{E}_{k}^{n}\right),
$$

where

$$
I_{m}=\frac{1}{\Delta x^{2}} \operatorname{Re} \sum_{k}\left(\bar{E}_{k}^{n+1}-\bar{E}_{k}^{n}\right)\left(E_{k+1}^{m}-2 E_{k}^{m}+E_{k-1}^{m}\right) \quad(m=n, n+1) .
$$

The right side of (18) equals

$$
\frac{1}{2} \sum_{k}\left(\left|E_{k}^{n+1}\right|^{2}-\left|E_{k}^{n}\right|^{2}\right)\left(N_{k}^{n+1}+N_{k}^{n}\right)
$$

Summing by parts, we get for the left side of (18)

$$
I_{n}+I_{n+1}=-\frac{1}{\Delta x^{2}} \sum_{k}\left|E_{k+1}^{n+1}-E_{k}^{n+1}\right|^{2}+\frac{1}{\Delta x^{2}} \sum_{k}\left|E_{k+1}^{n}-E_{k}^{n}\right|^{2} .
$$

Thus (19), (20) yield the identity

$$
-\sum_{k}\left|\delta E_{k}^{n+1}\right|^{2}+\sum_{k}\left|\delta E_{k}^{n}\right|^{2}=\frac{1}{2} \sum_{k}\left(\left|E_{k}^{n+1}\right|^{2}-\left|E_{k}^{n}\right|^{2}\right)\left(N_{k}^{n+1}+N_{k}^{n}\right) .
$$

We obtain the contribution from $\left\{N_{k}^{n}\right\}$ by recalling from Lemma 2 that

$$
\delta^{2} u_{k}^{n} \equiv \frac{u_{k+1}^{n}-2 u_{k}^{n}+u_{k-1}^{n}}{\Delta x^{2}}=\frac{N_{k}^{n+1}-N_{k}^{n}}{\Delta t}
$$

and by multiplying (8.N) by $\frac{1}{2}\left(u_{k}^{n}+u_{k}^{n-1}\right)$ and then summing on $k$. There results

$$
\mathrm{I}-\mathrm{II}=\mathrm{III},
$$


where

$$
\begin{aligned}
\mathrm{I} & =\frac{1}{2} \sum_{k} \frac{\left(N_{k}^{n+1}-2 N_{k}^{n}+N_{k}^{n-1}\right)}{\Delta t^{2}}\left(u_{k}^{n}+u_{k}^{n-1}\right), \\
\mathrm{II} & =\frac{1}{4} \sum_{k} \frac{\left(u_{k}^{n}+u_{k}^{n-1}\right)}{\Delta x^{2}}\left[N_{k+1}^{n+1}-2 N_{k}^{n+1}+N_{k-1}^{n+1}+N_{k+1}^{n-1}-2 N_{k}^{n-1}+N_{k-1}^{n-1}\right], \\
\mathrm{III} & =\frac{1}{2} \sum_{k} \frac{\left(u_{k}^{n}+u_{k}^{n-1}\right)}{\Delta x^{2}}\left[\left|E_{k+1}^{n}\right|^{2}-2\left|E_{k}^{n}\right|^{2}+\left|E_{k-1}^{n}\right|^{2}\right] .
\end{aligned}
$$

Term III is summed by parts:

$$
\begin{aligned}
\mathrm{III}= & -\frac{1}{2 \Delta x^{2}} \sum_{k}\left[\left(u_{k+1}^{n}+u_{k+1}^{n-1}\right)-\left(u_{k}^{n}+u_{k}^{n-1}\right)\right]\left[\left|E_{k+1}^{n}\right|^{2}-\left|E_{k}^{n}\right|^{2}\right] \\
= & -\frac{1}{2 \Delta x^{2}} \sum_{k}\left[u_{k}^{n}+u_{k}^{n-1}-u_{k-1}^{n}-u_{k-1}^{n-1}\right]\left|E_{k}^{n}\right|^{2} \\
& +\frac{1}{2 \Delta x^{2}} \sum_{k}\left[u_{k+1}^{n}+u_{k+1}^{n-1}-u_{k}^{n}-u_{k}^{n-1}\right]\left|E_{k}^{n}\right|^{2},
\end{aligned}
$$

where we have shifted $k \rightarrow k-1$ to obtain the first sum. Thus, by (22),

$$
\begin{aligned}
\mathrm{III} & =\frac{1}{2 \Delta x^{2}} \sum_{k}\left|E_{k}^{n}\right|^{2}\left[\left(u_{k+1}^{n}-2 u_{k}^{n}+u_{k-1}^{n}\right)+\left(u_{k+1}^{n-1}-2 u_{k}^{n-1}+u_{k-1}^{n-1}\right)\right] \\
& =\frac{1}{2} \sum_{k}\left|E_{k}^{n}\right|^{2}\left[\frac{N_{k}^{n+1}-N_{k}^{n}}{\Delta t}+\frac{N_{k}^{n}-N_{k}^{n-1}}{\Delta t}\right] \\
& =\frac{1}{2 \Delta t} \sum_{k}\left|E_{k}^{n}\right|^{2}\left(N_{k}^{n+1}-N_{k}^{n-1}\right) .
\end{aligned}
$$

To evaluate I, we note that by $(22)$

$$
\delta^{2} u_{k}^{n}-\delta^{2} u_{k}^{n-1}=\frac{N_{k}^{n+1}-N_{k}^{n}}{\Delta t}-\left(\frac{N_{k}^{n}-N_{k}^{n-1}}{\Delta t}\right)=\frac{N_{k}^{n+1}-2 N_{k}^{n}+N_{k}^{n-1}}{\Delta t}
$$

Thus,

$$
\mathrm{I}=\frac{1}{2 \Delta t} \sum_{k}\left(u_{k}^{n}+u_{k}^{n-1}\right)\left[\delta^{2} u_{k}^{n}-\delta^{2} u_{k}^{n-1}\right]
$$

and, summing this by parts, we get

$$
\mathrm{I}=-\frac{1}{2 \Delta t} \sum_{k}\left(\delta u_{k}^{n}\right)^{2}+\frac{1}{2 \Delta t} \sum_{k}\left(\delta u_{k}^{n-1}\right)^{2}
$$


Summing II now by parts, we find

$$
\begin{aligned}
\mathrm{II}= & -\frac{1}{4 \Delta x^{2}} \sum_{k}\left[\left(u_{k+1}^{n}+u_{k+1}^{n-1}\right)-\left(u_{k}^{n}+u_{k}^{n-1}\right)\right] \\
& \cdot\left[\left(N_{k+1}^{n+1}-N_{k}^{n+1}\right)+\left(N_{k+1}^{n-1}-N_{k}^{n-1}\right)\right] \\
= & -\frac{1}{4 \Delta x^{2}} \sum_{k}\left[u_{k}^{n}+u_{k}^{n-1}-u_{k-1}^{n}-u_{k-1}^{n-1}\right]\left[N_{k}^{n+1}+N_{k}^{n-1}\right] \\
& +\frac{1}{4 \Delta x^{2}} \sum_{k}\left[u_{k+1}^{n}+u_{k+1}^{n-1}-u_{k}^{n}-u_{k}^{n-1}\right]\left[N_{k}^{n+1}+N_{k}^{n-1}\right]
\end{aligned}
$$

where we have again shifted $k \rightarrow k-1$ to get the first sum. Thus, by (22),

$$
\begin{aligned}
\mathrm{II} & =\frac{1}{4 \Delta x^{2}} \sum_{k}\left(N_{k}^{n+1}+N_{k}^{n-1}\right)\left[\left(u_{k+1}^{n}-2 u_{k}^{n}+u_{k-1}^{n}\right)+\left(u_{k+1}^{n-1}-2 u_{k}^{n-1}+u_{k-1}^{n-1}\right)\right] \\
& =\frac{1}{4} \sum_{k}\left(N_{k}^{n+1}+N_{k}^{n-1}\right)\left[\frac{N_{k}^{n+1}-N_{k}^{n}}{\Delta t}+\frac{N_{k}^{n}-N_{k}^{n-1}}{\Delta t}\right] \\
& =\frac{1}{4 \Delta t} \sum_{k}\left[\left(N_{k}^{n+1}\right)^{2}-\left(N_{k}^{n-1}\right)^{2}\right] .
\end{aligned}
$$

Therefore, equation (23) yields

$$
\begin{aligned}
-\frac{1}{2 \Delta t} & \sum_{k}\left(\delta u_{k}^{n}\right)^{2}-\frac{1}{4 \Delta t} \sum_{k}\left(N_{k}^{n+1}\right)^{2} \\
= & -\frac{1}{2 \Delta t} \sum_{k}\left(\delta u_{k}^{n-1}\right)^{2}-\frac{1}{4 \Delta t} \sum_{k}\left(N_{k}^{n-1}\right)^{2} \\
& +\frac{1}{2 \Delta t} \sum_{k}\left|E_{k}^{n}\right|^{2}\left(N_{k}^{n+1}-N_{k}^{n-1}\right) .
\end{aligned}
$$

Now multiply this by $\Delta t$ and add the result to (21) to get

$$
\begin{aligned}
-\frac{1}{2} \sum_{k}\left(\delta u_{k}^{n}\right)^{2}-\frac{1}{4} \sum_{k}\left(N_{k}^{n+1}\right)^{2}-\sum_{k}\left|\delta E_{k}^{n+1}\right|^{2} \\
=-\frac{1}{2} \sum_{k}\left(\delta u_{k}^{n-1}\right)^{2}-\frac{1}{4} \sum_{k}\left(N_{k}^{n-1}\right)^{2}-\sum_{k}\left|\delta E_{k}^{n}\right|^{2} \\
\quad+\frac{1}{2} \sum_{k}\left[\left|E_{k}^{n}\right|^{2}\left(N_{k}^{n+1}-N_{k}^{n-1}\right)+\left(\left|E_{k}^{n+1}\right|^{2}-\left|E_{k}^{n}\right|^{2}\right)\left(N_{k}^{n+1}+N_{k}^{n}\right)\right]
\end{aligned}
$$

The last term here equals

$$
\frac{1}{2} \sum_{k}\left|E_{k}^{n+1}\right|^{2}\left(N_{k}^{n+1}+N_{k}^{n}\right)-\frac{1}{2} \sum_{k}\left|E_{k}^{n}\right|^{2}\left(N_{k}^{n}+N_{k}^{n-1}\right) .
$$

Therefore, when we define $\mathscr{E}_{d}^{n+1}$ as in part (b) of Theorem 1, (28) implies $\mathscr{E}_{d}^{n+1}=\mathscr{E}_{d}^{n}$ and hence $\mathscr{E}_{d}^{n}=\mathscr{E}_{d}^{0}$ and energy is conserved.

In order to state the main theorem, we define the errors by

$$
\begin{aligned}
& e_{k}^{n}=E\left(x_{k}, t^{n}\right)-E_{k}^{n}, \\
& \eta_{k}^{n}=N\left(x_{k}, t^{n}\right)-N_{k}^{n} .
\end{aligned}
$$


Here, $E_{k}^{n}, N_{k}^{n}$ are computed from the scheme (8.E), (8.N) for $n \Delta t \leq T, 1 \leq$ $k \leq J$.

Lemma 4. Let the data satisfy (H0). Define $\left\{U_{k}^{n}\right\}$ by

$$
\frac{U_{k+1}^{n}-2 U_{k}^{n}+U_{k-1}^{n}}{\Delta x^{2}}=\frac{\eta_{k}^{n+1}-\eta_{k}^{n}}{\Delta t}, \quad k=1, \ldots, J-1,
$$

Extend $\left\{U_{k}^{n}\right\}$ by defining

$$
U_{k}^{n}=U_{j}^{n} \quad \text { if } k \equiv j \bmod J
$$

Then

$$
U_{k}^{n}=-\Delta x \sum_{j=1}^{J-1} G\left(x_{k}, x_{j}\right) \frac{\eta_{j}^{n+1}-\eta_{j}^{n}}{\Delta t},
$$

where

$$
G(x, y)= \begin{cases}x\left(1-\frac{y}{L}\right), & 0 \leq x \leq y \leq L, \\ y\left(1-\frac{x}{L}\right), & 0 \leq y \leq x \leq L .\end{cases}
$$

Proof. The actual computation showing that the given representation is a solution is easy and is omitted. As in Lemma 2, there remains the compatibility question. Using the definition (30) of $\eta_{k}^{n}$, we have

$$
\begin{aligned}
\delta^{2} U_{k}^{n} & =\Delta t^{-1}\left[N\left(x_{k}, t^{n+1}\right)-N_{k}^{n+1}-N\left(x_{k}, t^{n}\right)+N_{k}^{n}\right] \\
& =-\delta^{2} u_{k}^{n}+\Delta t^{-1}\left[N\left(x_{k}, t^{n+1}\right)-N\left(x_{k}, t^{n}\right)\right] .
\end{aligned}
$$

Therefore, as in Lemma 2, we require that

$$
S \equiv \sum_{k=1}^{J}\left[N\left(x_{k}, t^{n+1}\right)-N\left(x_{k}, t^{n}\right)\right]=0 .
$$

We expand $N(x, t)$ in a Fourier series with Fourier coefficients $\left\{c_{m}\right\}$ :

$$
N(x, t)=\sum_{m} c_{m}(t) \exp \left(\frac{2 i m \pi x}{L}\right) .
$$

Thus, $c_{0}(t)$ is proportional to $\int_{0}^{L} N(x, t) d x$. Integrating (ZS. $\left.N\right)$ over a period, we see that this integral is a linear function of $t$. In fact, $c_{0}(t)$ is constant in time in view of $(\mathrm{H} 0)$. Now we write

$$
\sum_{k=1}^{J} N\left(x_{k}, t\right)=\sum_{m} c_{m}(t) \sum_{k=1}^{J} \exp \left(\frac{2 i m \pi x_{k}}{L}\right)
$$

and evaluate the inner sum explicitly. Using $x_{k}=k \Delta x=k L / J$, we see that this sum over $k$ vanishes unless $m=0$, in which case

$$
\sum_{k=1}^{J} N\left(x_{k}, t\right)=J c_{0}(t)
$$

Hence $S=0$ as desired.

The norms are defined, e.g., as $\left\|e^{n}\right\|_{2}^{2}=\sum_{k=1}^{J}\left|e_{k}^{n}\right|^{2} \Delta x$, etc. 
Theorem 2. Let $T>0$; assume (HE) and that the data satisfy (H0). Given any positive integer $J$, let $J \Delta x=L$ and choose $\Delta t=\Delta x$. Let $E_{k}^{n}, N_{k}^{n}$ be computed from the scheme (8.E), (8.N), (9), (10) for $n \Delta t \leq T$. Define

$$
\mathscr{E}^{n}=\frac{1}{2}\left[\left\|e^{n+1}\right\|_{2}^{2}+\left\|\delta e^{n+1}\right\|_{2}^{2}+\left\|\delta U^{n}\right\|_{2}^{2}+\frac{1}{2}\left(\left\|\eta^{n+1}\right\|_{2}^{2}+\left\|\eta^{n}\right\|_{2}^{2}\right)\right] .
$$

(Thus, $\mathscr{E}^{n}$ is the (square of the) "energy norm" of the errors.)

Then there exists a constant $c_{T}$ depending only on the data and $T$, with the property that for $\Delta x$ sufficiently small, we have

$$
\mathscr{E}^{n} \leq c_{T}\left[\mathscr{E}^{0}+\Delta x^{2}\right] .
$$

Moreover, $\mathscr{E}^{0}=O\left(\Delta x^{2}\right)$, and hence

$$
\mathscr{E}^{n} \leq c_{T} \Delta x^{2} \text { as } \Delta x \rightarrow 0 .
$$

The proof of Theorem 2 will be given in the next section.

Remark. The choice $\Delta t=\Delta x$ allows us to easily combine several estimates. It is seen from the proof that the same estimates can be obtained provided $\Delta t$ is bounded both above and below by a constant times $\Delta x$.

3. CoNVERgence estimates, PROOF OF THE MAIN THEOREM

We begin by defining the standard discretization errors

$$
\begin{aligned}
\tau_{k}^{n}= & \frac{i}{\Delta t}\left(E\left(x_{k}, t^{n+1}\right)-E\left(x_{k}, t^{n}\right)\right) \\
& +\frac{1}{2 \Delta x^{2}}\left(E\left(x_{k+1}, t^{n}\right)-2 E\left(x_{k}, t^{n}\right)+E\left(x_{k-1}, t^{n}\right)\right) \\
& +\frac{1}{2 \Delta x^{2}}\left(E\left(x_{k+1}, t^{n+1}\right)-2 E\left(x_{k}, t^{n+1}\right)+E\left(x_{k-1}, t^{n+1}\right)\right) \\
& -\frac{1}{4}\left(N\left(x_{k}, t^{n}\right)+N\left(x_{k}, t^{n+1}\right)\right)\left(E\left(x_{k}, t^{n}\right)+E\left(x_{k}, t^{n+1}\right)\right)
\end{aligned}
$$

and

$$
\begin{aligned}
\sigma_{k}^{n}= & \frac{1}{\Delta t^{2}}\left(N\left(x_{k}, t^{n+1}\right)-2 N\left(x_{k}, t^{n}\right)+N\left(x_{k}, t^{n-1}\right)\right) \\
& -\frac{1}{2 \Delta x^{2}}\left(N\left(x_{k+1}, t^{n+1}\right)-2 N\left(x_{k}, t^{n+1}\right)+N\left(x_{k-1}, t^{n+1}\right)\right) \\
& -\frac{1}{2 \Delta x^{2}}\left(N\left(x_{k+1}, t^{n-1}\right)-2 N\left(x_{k}, t^{n-1}\right)+N\left(x_{k-1}, t^{n-1}\right)\right) \\
& -\frac{1}{\Delta x^{2}}\left(\left|E\left(x_{k+1}, t^{n}\right)\right|^{2}-2\left|E\left(x_{k}, t^{n}\right)\right|^{2}+\left|E\left(x_{k-1}, t^{n}\right)\right|^{2}\right) .
\end{aligned}
$$

As usual, these measure the amount by which the exact solutions fail to satisfy the approximate equations.

Recall that $E, N$ are smooth solutions.

Lemma 5. We have $\left|\tau_{k}^{n}\right|+\left|\sigma_{k}^{n}\right|=O\left(\Delta t^{2}+\Delta x^{2}\right)$ as $\Delta x, \Delta t \rightarrow 0$.

Proof. By Taylor's theorem and (ZS.E) we can write the first three terms $\tau_{3}$ 
in $\tau_{k}^{n}$ as

$$
\begin{aligned}
\tau_{3}= & i\left(E_{t}\left(x_{k}, t^{n}\right)+\frac{1}{2} \Delta t E_{t t}\left(x_{k}, \beta_{k}^{n}\right)\right)+\frac{1}{2}\left(E_{x x}\left(x_{k}, t^{n}\right)+O\left(\Delta x^{2}\right)\right) \\
& +\frac{1}{2}\left(E_{x x}\left(x_{k}, t^{n+1}\right)+O\left(\Delta x^{2}\right)\right) \quad\left(t^{n}<\beta_{k}^{n}<t^{n+1}\right) \\
= & i E_{t}\left(x_{k}, t^{n}\right)+\frac{i \Delta t}{2} E_{t t}\left(x_{k}, \beta_{k}^{n}\right)+O\left(\Delta x^{2}\right) \\
& +\frac{1}{2}\left[N\left(x_{k}, t^{n}\right) E\left(x_{k}, t^{n}\right)-i E_{t}\left(x_{k}, t^{n}\right)\right] \\
& \quad+\frac{1}{2}\left[N\left(x_{k}, t^{n+1}\right) E\left(x_{k}, t^{n+1}\right)-i E_{t}\left(x_{k}, t^{n+1}\right)\right] \\
= & \frac{N\left(x_{k}, t^{n}\right) E\left(x_{k}, t^{n}\right)+N\left(x_{k}, t^{n+1}\right) E\left(x_{k}, t^{n+1}\right)}{2}+O\left(\Delta x^{2}\right) \\
& \quad+\frac{i \Delta t}{2} E_{t t}\left(x_{k}, \beta_{k}^{n}\right)+\frac{i}{2}\left[E_{t}\left(x_{k}, t^{n}\right)-E_{t}\left(x_{k}, t^{n+1}\right)\right] \\
= & \frac{N\left(x_{k}, t^{n}\right) E\left(x_{k}, t^{n}\right)+N\left(x_{k}, t^{n+1}\right) E\left(x_{k}, t^{n+1}\right)}{2}+O\left(\Delta t^{2}+\Delta x^{2}\right) .
\end{aligned}
$$

Now the result for $\tau_{k}^{n}$ will follow if

$$
\begin{aligned}
& \frac{1}{2}\left(N\left(x_{k}, t^{n}\right) E\left(x_{k}, t^{n}\right)+N\left(x_{k}, t^{n+1}\right) E\left(x_{k}, t^{n+1}\right)\right) \\
& \quad-\frac{1}{4}\left(N\left(x_{k}, t^{n}\right)+N\left(x_{k}, t^{n+1}\right)\right)\left(E\left(x_{k}, t^{n}\right)+E\left(x_{k}, t^{n+1}\right)\right) \\
& =O\left(\Delta t^{2}+\Delta x^{2}\right) .
\end{aligned}
$$

Simple algebra shows that this expression equals

$$
\frac{1}{4}\left(E\left(x_{k}, t^{n+1}\right)-E\left(x_{k}, t^{n}\right)\right)\left(N\left(x_{k}, t^{n+1}\right)-N\left(x_{k}, t^{n}\right)\right),
$$

and hence is $O\left(\Delta t^{2}\right)$.

As for $\sigma_{k}^{n}$, we use Taylor's theorem again to write

$$
\begin{aligned}
\sigma_{k}^{n}= & \left(N_{t t}\left(x_{k}, t^{n}\right)+O\left(\Delta t^{2}\right)\right)-\frac{1}{2}\left(N_{x x}\left(x_{k}, t^{n+1}\right)+O\left(\Delta x^{2}\right)\right) \\
& -\frac{1}{2}\left(N_{x x}\left(x_{k}, t^{n-1}\right)+O\left(\Delta x^{2}\right)\right)-\left(\frac{\partial^{2}}{\partial x^{2}}\left|E\left(x_{k}, t^{n}\right)\right|^{2}+O\left(\Delta x^{2}\right)\right) .
\end{aligned}
$$

The result follows from (ZS.N), since

$$
N_{x x}\left(x_{k}, t^{n}\right)-\frac{1}{2}\left(N_{x x}\left(x_{k}, t^{n+1}\right)+N_{x x}\left(x_{k}, t^{n-1}\right)\right)=O\left(\Delta t^{2}\right) .
$$

Recall that the errors are defined by (29), (30). In order to obtain the error equations we subtract (8.E) from the definition (34) of $\tau_{k}^{n}$ to get

$$
\begin{gathered}
i\left(\frac{e_{k}^{n+1}-e_{k}^{n}}{\Delta t}\right)+\frac{1}{2} \delta^{2} e_{k}^{n}+\frac{1}{2} \delta^{2} e_{k}^{n+1} \\
=\tau_{k}^{n}+\frac{1}{4}\left[N\left(x_{k}, t^{n}\right)+N\left(x_{k}, t^{n+1}\right)\right]\left[E\left(x_{k}, t^{n}\right)+E\left(x_{k}, t^{n+1}\right)\right] \\
\quad-\frac{1}{4}\left[N_{k}^{n}+N_{k}^{n+1}\right]\left[E_{k}^{n}+E_{k}^{n+1}\right] \\
=\tau_{k}^{n}+\frac{1}{4}\left[\left(\eta_{k}^{n}+\eta_{k}^{n+1}\right)\left(E\left(x_{k}, t^{n}\right)+E\left(x_{k}, t^{n+1}\right)\right)\right. \\
\left.+\left(N_{k}^{n}+N_{k}^{n+1}\right)\left(e_{k}^{n}+e_{k}^{n+1}\right)\right] .
\end{gathered}
$$


Subtracting (8.N) from (35), the definition of $\sigma_{k}^{n}$, we get similarly

$$
\begin{gathered}
\frac{\eta_{k}^{n+1}-2 \eta_{k}^{n}+\eta_{k}^{n-1}}{\Delta t^{2}}-\frac{1}{2} \delta^{2} \eta_{k}^{n+1}-\frac{1}{2} \delta^{2} \eta_{k}^{n-1} \\
=\sigma_{k}^{n}+\delta^{2}\left(\left|E\left(x_{k}, t^{n}\right)\right|^{2}-\left|E_{k}^{n}\right|^{2}\right) .
\end{gathered}
$$

In a sequence of lemmas we will derive energy estimates on $e$ and $\eta$.

Lemma 6 ( $L^{2}$-estimate of $e$ ). There are constants $c, c_{T}$ such that for $\Delta x, \Delta t$ sufficiently small,

$$
\begin{aligned}
\left\|e^{n+1}\right\|_{2}^{2} \leq & (1+c \Delta t)\left\|e^{n}\right\|_{2}^{2}+c_{T}\left(\Delta t^{2}+\Delta x^{2}\right)^{2} \Delta t \\
& +c \Delta t\left(\left\|\eta^{n+1}\right\|_{2}^{2}+\left\|\eta^{n}\right\|_{2}^{2}\right) .
\end{aligned}
$$

Proof. As in Theorem 1(a), we multiply (36) by $\bar{e}_{k}^{n+1}+\bar{e}_{k}^{n}$, sum on $k$, and take the imaginary part to get

$$
\mathrm{I}+\mathrm{II}=\mathrm{III}+\mathrm{IV},
$$

where

$$
\begin{aligned}
\mathrm{I} & =\frac{1}{\Delta t} \operatorname{Re} \sum_{k}\left(e_{k}^{n+1}-e_{k}^{n}\right)\left(\bar{e}_{k}^{n+1}+\bar{e}_{k}^{n}\right)=\frac{1}{\Delta t} \sum_{k}\left(\left|e_{k}^{n+1}\right|^{2}-\left|e_{k}^{n}\right|^{2}\right), \\
\mathrm{II} & =\frac{1}{2} \operatorname{Im} \sum_{k}\left(\bar{e}_{k}^{n+1}+\bar{e}_{k}^{n}\right)\left(\delta^{2} e_{k}^{n+1}+\delta^{2} e_{k}^{n}\right), \\
\mathrm{III} & =\operatorname{Im} \sum_{k}\left(\bar{e}_{k}^{n+1}+\bar{e}_{k}^{n}\right) \tau_{k}^{n}, \\
\mathrm{IV} & =\frac{1}{4} \operatorname{Im} \sum_{k}\left(\bar{e}_{k}^{n+1}+\bar{e}_{k}^{n}\right)\left[\left(\eta_{k}^{n}+\eta_{k}^{n+1}\right)\left(E\left(x_{k}, t^{n}\right)+E\left(x_{k}, t^{n+1}\right)\right)\right],
\end{aligned}
$$

the last simplifying since $N$ is real. All sums are taken over indices $k$ with $1 \leq k \leq J$.

Term $\mathrm{I}$ is as desired. For III, we have from Lemma 5

$$
\begin{aligned}
|\mathrm{III}| & \leq c \sum_{k}\left(\left|e_{k}^{n+1}\right|^{2}+\left|e_{k}^{n}\right|^{2}\right)+c \sum_{k}\left|\tau_{k}^{n}\right|^{2} \\
& \leq c \Delta x^{-1}\left(\left\|e^{n+1}\right\|_{2}^{2}+\left\|e^{n}\right\|_{2}^{2}\right)+c_{T}\left(\Delta t^{2}+\Delta x^{2}\right)^{2} \cdot J,
\end{aligned}
$$

and IV is easily estimable by

$$
\begin{aligned}
|\mathrm{IV}| & \leq c \sup _{x, t \leq T}|E(x, t)| \cdot \sum_{k} \frac{\left(\left|e_{k}^{n+1}\right|+\left|e_{k}^{n}\right|\right) \Delta x^{1 / 2} \cdot\left(\left|\eta_{k}^{n+1}\right|+\left|\eta_{k}^{n}\right|\right) \Delta x^{1 / 2}}{\Delta x} \\
& \leq c \Delta x^{-1}\left[\left\|e^{n+1}\right\|_{2}^{2}+\left\|e^{n}\right\|_{2}^{2}+\left\|\eta^{n+1}\right\|_{2}^{2}+\left\|\eta^{n}\right\|_{2}^{2}\right] .
\end{aligned}
$$

As before, term II vanishes upon summation by parts. Now we multiply (38) by $\Delta t \Delta x$ and use the bounds derived above to get

$$
\begin{gathered}
\left\|e^{n+1}\right\|_{2}^{2} \leq\left\|e^{n}\right\|_{2}^{2}+c \Delta t\left(\left\|e^{n+1}\right\|_{2}^{2}+\left\|e^{n}\right\|_{2}^{2}\right)+c_{T}\left(\Delta t^{2}+\Delta x^{2}\right)^{2} \cdot J \Delta t \Delta x \\
+c \Delta t\left(\left\|e^{n+1}\right\|_{2}^{2}+\left\|e^{n}\right\|_{2}^{2}+\left\|\eta^{n+1}\right\|_{2}^{2}+\left\|\eta^{n}\right\|_{2}^{2}\right) .
\end{gathered}
$$

Thus, we have

$$
\begin{aligned}
(1-c \Delta t)\left\|e^{n+1}\right\|_{2}^{2} \leq & (1+c \Delta t)\left\|e^{n}\right\|_{2}^{2}+c_{T}\left(\Delta t^{2}+\Delta x^{2}\right)^{2} \Delta t \\
& +c \Delta t\left(\left\|\eta^{n+1}\right\|_{2}^{2}+\left\|\eta^{n}\right\|_{2}^{2}\right),
\end{aligned}
$$

and the result follows. 
When estimating the energy, we will need bounds on the discrete potentials $u_{k}^{n}$ from Lemma 2 and $U_{k}^{n}$ from Lemma 4.

Lemma 7. There is a constant $c$ depending only on the data such that

$$
\sup _{k}\left|u_{k}^{n}\right| \leq c
$$

Proof. We write, using the boundary condition $u_{0}^{n}=0$,

$$
\left|u_{k}^{n}\right|=\left|\sum_{j=1}^{k}\left(u_{j}^{n}-u_{j-1}^{n}\right)\right|=\left|\Delta x \sum_{j=1}^{k} \delta u_{j-1}^{n}\right| \leq\left\|\delta u^{n}\right\|_{2}(J \Delta x)^{1 / 2},
$$

and this is bounded by Lemma 3 and the definition of $J$.

Lemma 8. Let $U_{k}^{n}$ be defined as in Lemma 4. There is a constant $c$ such that

$$
\sup _{k}\left|U_{k}^{n}\right| \leq c\left(\mathscr{C}^{n}\right)^{1 / 2}
$$

Proof. The proof is the same as that of Lemma 7, but in the last step we use the definition of $\mathscr{E}^{n}$ from Theorem 2.

Lemma 9 (Energy of $e)$. Let $h=\Delta t=\Delta x$, and define

$$
\begin{aligned}
\mathrm{II}^{n} & =\frac{1}{2} \operatorname{Re} \sum_{k}\left(E\left(x_{k}, t^{n}\right)+E\left(x_{k}, t^{n+1}\right)\right)\left(\eta_{k}^{n+1}+\eta_{k}^{n}\right) \bar{e}_{k}^{n+1}, \\
\mathrm{III}^{n} & =\frac{1}{2} \sum_{k}\left(N_{k}^{n+1}+N_{k}^{n}\right)\left|e_{k}^{n+1}\right|^{2} .
\end{aligned}
$$

Then

$$
\begin{aligned}
& \frac{1}{2}\left\|\delta e^{n}\right\|_{2}^{2}+h\left(\mathrm{II}^{n-1}+\mathrm{III}^{n-1}\right)-\left(\frac{1}{2}\left\|\delta e^{n+1}\right\|_{2}^{2}+h\left(\mathrm{II}^{n}+\mathrm{III}^{n}\right)\right) \\
& \quad=O\left[h\left(\mathscr{E}^{n}+\mathscr{E}^{n-1}\right)+h^{3}\right] .
\end{aligned}
$$

Proof. As in Theorem 1(b), we multiply (36) by $\left(\bar{e}_{k}^{n+1}-\bar{e}_{k}^{n}\right)$, sum over $k$, $k=1, \ldots, J$, add the result to its conjugate, and take the real part. There results the identity

$$
\mathrm{I}_{0}=\mathrm{I}+\mathrm{II}+\mathrm{III} \text {, }
$$

where

$$
\begin{aligned}
\mathrm{I}_{0} & =\operatorname{Re} \sum_{k}\left(\bar{e}_{k}^{n+1}-\bar{e}_{k}^{n}\right)\left(\delta^{2} e_{k}^{n}+\delta^{2} e_{k}^{n+1}\right), \\
|\mathrm{I}| & =\left|2 \operatorname{Re} \sum_{k} \tau_{k}^{n}\left(\bar{e}_{k}^{n+1}-\bar{e}_{k}^{n}\right)\right| \\
& \leq c_{T} h^{2} J^{1 / 2} h^{-1 / 2}\left(\left\|e^{n+1}\right\|_{2}+\left\|e^{n}\right\|_{2}\right) \leq c_{T} h\left(\mathscr{E}^{n}+\mathscr{E}^{n-1}\right)^{1 / 2}, \\
\mathrm{II} & =\frac{1}{2} \operatorname{Re} \sum_{k}\left(\eta_{k}^{n+1}+\eta_{k}^{n}\right)\left(E\left(x_{k}, t^{n}\right)+E\left(x_{k}, t^{n+1}\right)\right)\left(\bar{e}_{k}^{n+1}-\bar{e}_{k}^{n}\right), \\
\mathrm{III} & =\frac{1}{2} \sum_{k}\left(N_{k}^{n}+N_{k}^{n+1}\right)\left(\left|e_{k}^{n+1}\right|^{2}-\left|e_{k}^{n}\right|^{2}\right) .
\end{aligned}
$$


We sum $I_{0}$ by parts to get

$$
\mathrm{I}_{0}=\frac{1}{2} \sum_{k}\left|\delta e_{k}^{n}\right|^{2}-\frac{1}{2} \sum_{k}\left|\delta e_{k}^{n+1}\right|^{2} .
$$

Next, we rewrite term III as

$$
\begin{aligned}
\mathrm{III} & =\frac{1}{2} \sum_{k}\left[\left(N_{k}^{n+1}+N_{k}^{n}\right)\left|e_{k}^{n+1}\right|^{2}-\left(N_{k}^{n}+N_{k}^{n-1}\right)\left|e_{k}^{n}\right|^{2}+\left(N_{k}^{n-1}-N_{k}^{n+1}\right)\left|e_{k}^{n}\right|^{2}\right] \\
& \equiv \mathrm{III}^{n}-\mathrm{III}^{n-1}+\frac{1}{2} \sum_{k}\left(N_{k}^{n-1}-N_{k}^{n+1}\right)\left|e_{k}^{n}\right|^{2}
\end{aligned}
$$

where

$$
\mathrm{III}^{n}=\frac{1}{2} \sum_{k}\left(N_{k}^{n+1}+N_{k}^{n}\right)\left|e_{k}^{n+1}\right|^{2} .
$$

Recall from the definition (Lemma 2) of $u_{k}^{n}$ that

$$
\delta^{2} u_{k}^{n}=\frac{N_{k}^{n+1}-N_{k}^{n}}{h}
$$

Thus,

and therefore

$$
\delta^{2}\left(u_{k}^{n}+u_{k}^{n-1}\right)=\frac{N_{k}^{n+1}-N_{k}^{n-1}}{h}
$$

$$
\mathrm{III}=\mathrm{III}^{n}-\mathrm{III}^{n-1}-\frac{1}{2} h \sum_{k}\left|e_{k}^{n}\right|^{2} \delta^{2}\left(u_{k}^{n}+u_{k}^{n-1}\right) .
$$

We sum by parts to get for the last term the bound

$$
\begin{aligned}
O\left(h \sum_{k}\left|e_{k}^{n} \| \delta e_{k}^{n}\right|\left(\left|\delta u_{k}^{n}\right|+\left|\delta u_{k}^{n-1}\right|\right)\right) & =O\left(\left\|e^{n}\right\|_{\infty}\left\|\delta e^{n}\right\|_{2}\left(\left\|\delta u^{n}\right\|_{2}+\left\|\delta u^{n-1}\right\|_{2}\right)\right) \\
& =O\left(\left\|e^{n}\right\|_{2}^{1 / 2}\left\|\delta e^{n}\right\|_{2}^{3 / 2}\right),
\end{aligned}
$$

where we have used Lemma 3. Hence,

$$
\mathrm{III}=\mathrm{III}^{n}-\mathrm{III}^{n-1}+O\left(\mathscr{E}^{n-1}\right) .
$$

Consider now term II. For brevity we set

$$
w_{k}^{n}=E\left(x_{k}, t^{n}\right)+E\left(x_{k}, t^{n+1}\right),
$$

so that

$$
w_{k}^{n}-w_{k}^{n-1}=E\left(x_{k}, t^{n+1}\right)-E\left(x_{k}, t^{n-1}\right)=O(h) .
$$

We write term II as

$$
\begin{aligned}
\mathrm{II}= & \frac{1}{2} \operatorname{Re} \sum_{k}\left(\eta_{k}^{n+1}+\eta_{k}^{n}\right) w_{k}^{n}\left(\bar{e}_{k}^{n+1}-\bar{e}_{k}^{n}\right) \\
= & \frac{1}{2} \operatorname{Re} \sum_{k} w_{k}^{n} \eta_{k}^{n+1} \bar{e}_{k}^{n+1}-\frac{1}{2} \operatorname{Re} \sum_{k} w_{k}^{n-1} \eta_{k}^{n} \bar{e}_{k}^{n}-\frac{1}{2} \operatorname{Re} \sum_{k}\left(w_{k}^{n}-w_{k}^{n-1}\right) \eta_{k}^{n} \bar{e}_{k}^{n} \\
& +\frac{1}{2} \operatorname{Re} \sum_{k} w_{k}^{n} \eta_{k}^{n} \bar{e}_{k}^{n+1}-\frac{1}{2} \operatorname{Re} \sum_{k} w_{k}^{n} \eta_{k}^{n+1} \bar{e}_{k}^{n} .
\end{aligned}
$$


Now we add and subtract the expression

$$
\frac{1}{2} \operatorname{Re} \sum_{k} w_{k}^{n-1} \eta_{k}^{n-1} \bar{e}_{k}^{n}
$$

and define

$$
\mathrm{II}^{n}=\frac{1}{2} \operatorname{Re} \sum_{k} w_{k}^{n} \eta_{k}^{n+1} e_{k}^{n+1}+\frac{1}{2} \operatorname{Re} \sum_{k} w_{k}^{n} \eta_{k}^{n} e_{k}^{n+1}
$$

Then, using Lemma 4, we can write II as

$$
\begin{aligned}
\mathrm{II}= & \mathrm{II}^{n}-\mathrm{II}^{n-1}+O\left(\mathscr{E}^{n-1}\right) \\
& -\frac{1}{2} \operatorname{Re} \sum_{k} \bar{e}_{k}^{n}\left[\left(w_{k}^{n}-w_{k}^{n-1}\right) \eta_{k}^{n+1}+w_{k}^{n-1}\left(\eta_{k}^{n+1}-\eta_{k}^{n-1}\right)\right] \\
= & \mathrm{II}^{n}-\mathrm{II}^{n-1}+O\left(\mathscr{E}^{n-1}\right)+O\left(\left(\mathscr{E}^{n-1}\right)^{1 / 2}\left(\mathscr{E}^{n}\right)^{1 / 2}\right) \\
& -\frac{1}{2} \operatorname{Re} \sum_{k} h \bar{e}_{k}^{n} w_{k}^{n-1} \delta^{2}\left(U_{k}^{n}+U_{k}^{n-1}\right) .
\end{aligned}
$$

We sum the last term here once by parts; it equals

$$
\begin{aligned}
& \frac{1}{2} \operatorname{Re} \sum_{k} h \delta\left(U_{k}^{n}+U_{k}^{n-1}\right)\left(w_{k}^{n-1} \delta e_{k}^{n}+\bar{e}_{k+1}^{n} \delta w_{k}^{n-1}\right) \\
& \quad=O\left[\left(\left\|\delta U^{n}\right\|_{2}+\left\|\delta U^{n-1}\right\|_{2}\right)\left(\left\|E\left(t^{n-1}\right)\right\|_{\infty}\left\|\delta e^{n}\right\|_{2}+\left\|E_{x}\left(t^{n-1}\right)\right\|_{\infty}\left\|e^{n}\right\|_{2}\right)\right] \\
& \quad=O\left(\left(\mathscr{E}^{n}+\mathscr{E}^{n-1}\right)\right) .
\end{aligned}
$$

Using these estimates in (46), we have

$$
\mathrm{II}=\mathrm{II}^{n}-\mathrm{II}^{n-1}+O\left[\mathscr{E}^{n}+\mathscr{E}^{n-1}\right]
$$

Finally, we multiply the relation

$$
\mathrm{I}_{0}=\mathrm{I}+\mathrm{II}+\mathrm{III}
$$

by $h$ and use the estimates for each of these terms derived above to get

$$
\begin{aligned}
\frac{1}{2}\left\|\delta e^{n}\right\|_{2}^{2}-\frac{1}{2}\left\|\delta e^{n+1}\right\|_{2}^{2}= & O\left(h^{3}\right)+O\left[h\left(\mathscr{E}^{n}+\mathscr{E}^{n-1}\right)\right] \\
& +\mathrm{II}^{n} h+\mathrm{III}^{n} h-\mathrm{II}^{n-1} h-\mathrm{III}^{n-1} h,
\end{aligned}
$$

or

$$
\begin{aligned}
& \frac{1}{2}\left\|\delta e^{n}\right\|_{2}^{2}+h\left(\mathrm{II}^{n-1}+\mathrm{III}^{n-1}\right)-\left(\frac{1}{2}\left\|\delta e^{n+1}\right\|_{2}^{2}+h\left(\mathrm{II}^{n}+\mathrm{III}^{n}\right)\right) \\
& =O\left(h\left(\mathscr{E}^{n}+\mathscr{E}^{n-1}\right)+h^{3}\right),
\end{aligned}
$$

and this is the statement of Lemma 9.

Lemma 10 ( $\eta$-energy). Let $h=\Delta t=\Delta x$. Then

$$
\begin{aligned}
& -\frac{1}{2}\left\|\delta U^{n}\right\|_{2}^{2}-\frac{1}{4}\left(\left\|\eta^{n+1}\right\|_{2}^{2}+\left\|\eta^{n}\right\|_{2}^{2}\right)+\frac{1}{2}\left\|\delta U^{n-1}\right\|_{2}^{2}+\frac{1}{4}\left(\left\|\eta^{n}\right\|_{2}^{2}+\left\|\eta^{n-1}\right\|_{2}^{2}\right) \\
& =O\left(h^{5}+h\left(\mathscr{E}^{n}+\mathscr{C}^{n-1}\right)\right) .
\end{aligned}
$$

Proof. Recall from Lemma 4 the relation

$$
\delta^{2} U_{k}^{n}=\frac{U_{k+1}^{n}-2 U_{k}^{n}+U_{k-1}^{n}}{h^{2}}=\frac{\eta_{k}^{n+1}-\eta_{k}^{n}}{h}
$$


We multiply the $\eta$-equation (37) by $\frac{1}{2}\left(U_{k}^{n}+U_{k}^{n-1}\right)$ and sum over $k$ to get the identity

$$
I_{1}-I_{2}-I_{3}=I_{4}+I_{5}
$$

where

$$
\begin{aligned}
& I_{1}=\frac{1}{2} \sum_{k}\left(U_{k}^{n}+U_{k}^{n-1}\right) \frac{\left(\eta_{k}^{n+1}-2 \eta_{k}^{n}+\eta_{k}^{n-1}\right)}{h^{2}}, \\
& I_{2}=\frac{1}{4} \sum_{k}\left(U_{k}^{n}+U_{k}^{n-1}\right) \delta^{2} \eta_{k}^{n-1}, \\
& I_{3}=\frac{1}{4} \sum_{k}\left(U_{k}^{n}+U_{k}^{n-1}\right) \delta^{2} \eta_{k}^{n+1}, \\
& I_{4}=\frac{1}{2} \sum_{k} \sigma_{k}^{n}\left(U_{k}^{n}+U_{k}^{n-1}\right)=O\left(h\left(\mathscr{E}^{n}+\mathscr{E}^{n-1}\right)^{1 / 2}\right) \quad \text { (by Lemma 8), } \\
& I_{5}=\frac{1}{2} \sum_{k}\left(U_{k}^{n}+U_{k}^{n-1}\right) \delta^{2}\left\{\left|E\left(x_{k}, t^{n}\right)\right|^{2}-\left|E_{k}^{n}\right|^{2}\right\} .
\end{aligned}
$$

We sum $I_{2}+I_{3}$ by parts, with the result

$$
I_{2}+I_{3}=-\frac{1}{4} \sum_{k} \delta\left(\eta_{k}^{n+1}+\eta_{k}^{n-1}\right) \delta\left(U_{k}^{n}+U_{k}^{n-1}\right) .
$$

Expansion of this yields

$$
\begin{aligned}
-\frac{1}{4 h^{2}} & \sum_{k}\left(\eta_{k+1}^{n+1}+\eta_{k+1}^{n-1}-\eta_{k}^{n+1}-\eta_{k}^{n-1}\right)\left(U_{k+1}^{n}+U_{k+1}^{n-1}-U_{k}^{n}-U_{k}^{n-1}\right) \\
= & -\frac{1}{4 h^{2}} \sum_{k}\left(\eta_{k}^{n+1}+\eta_{k}^{n-1}\right)\left(U_{k}^{n}+U_{k}^{n-1}-U_{k-1}^{n}-U_{k-1}^{n-1}\right) \\
& +\frac{1}{4 h^{2}} \sum_{k}\left(\eta_{k}^{n+1}+\eta_{k}^{n-1}\right)\left(U_{k+1}^{n}+U_{k+1}^{n-1}-U_{k}^{n}-U_{k}^{n-1}\right),
\end{aligned}
$$

where we put $k \rightarrow k-1$ to get the first sum. Thus,

$$
\begin{aligned}
I_{2}+I_{3} & =\frac{1}{4 h^{2}} \sum_{k}\left(\eta_{k}^{n+1}+\eta_{k}^{n-1}\right)\left[U_{k+1}^{n}-2 U_{k}^{n}+U_{k-1}^{n}+U_{k+1}^{n-1}-2 U_{k}^{n-1}+U_{k-1}^{n-1}\right] \\
& =\frac{1}{4} \sum_{k}\left(\eta_{k}^{n+1}+\eta_{k}^{n-1}\right)\left[\delta^{2} U_{k}^{n}+\delta^{2} U_{k}^{n-1}\right] \\
& =\frac{1}{4 h} \sum_{k}\left(\eta_{k}^{n+1}+\eta_{k}^{n-1}\right)\left[\left(\eta_{k}^{n+1}-\eta_{k}^{n}\right)+\left(\eta_{k}^{n}-\eta_{k}^{n-1}\right)\right] \\
& =\frac{1}{4 h} \sum_{k}\left(\left(\eta_{k}^{n+1}\right)^{2}-\left(\eta_{k}^{n-1}\right)^{2}\right) \\
& =\frac{1}{4 h} \sum_{k}\left(\left(\eta_{k}^{n+1}\right)^{2}+\left(\eta_{k}^{n}\right)^{2}\right)-\frac{1}{4 h} \sum_{k}\left(\left(\eta_{k}^{n}\right)^{2}+\left(\eta_{k}^{n-1}\right)^{2}\right) .
\end{aligned}
$$


Term $I_{5}$ is summed once by parts, with the result

$$
\begin{aligned}
& I_{5}=-\frac{1}{2 h^{2}} \sum_{k}\left(U_{k+1}^{n}+U_{k+1}^{n-1}-U_{k}^{n}-U_{k}^{n-1}\right) \\
& \cdot\left(\left|E\left(x_{k+1}, t^{n}\right)\right|^{2}-\left|E_{k+1}^{n}\right|^{2}-\left|E\left(x_{k}, t^{n}\right)\right|^{2}+\left|E_{k}^{n}\right|^{2}\right),
\end{aligned}
$$

and further expansion yields

$$
\begin{aligned}
& I_{5}=-\frac{1}{2 h} \operatorname{Re} \sum_{k}\left(\delta U_{k}^{n}+\delta U_{k}^{n-1}\right)\left.\quad-\left(E\left(x_{k}, t^{n}\right)-E_{k}^{n}\right)\left(\bar{E}\left(x_{k}, t^{n}\right)+\bar{E}_{k}^{n}\right)\right] \\
& \quad\left[\left(x_{k+1}, t^{n}\right)-E_{k+1}^{n}\right)\left(\bar{E}\left(x_{k+1}, t^{n}\right)+\bar{E}_{k+1}^{n}\right) \\
&=-\frac{1}{2 h} \operatorname{Re} \sum_{k}\left(\delta U_{k}^{n}+\delta U_{k}^{n-1}\right)\left.+e_{k}^{n}\left(\bar{E}\left(x_{k+1}, t^{n}\right)-\bar{E}\left(x_{k}, t^{n}\right)+\bar{E}_{k+1}^{n}-\bar{E}_{k}^{n}\right)\right] \\
&=-\frac{1}{2 h} \operatorname{Re} \sum_{k}\left(\delta U_{k}^{n}+\delta U_{k}^{n-1}\right)\left[\left(e_{k+1}^{n}-e_{k}^{n}\right)\left(\bar{E}\left(x_{k+1}, t^{n}\right)+\bar{E}_{k+1}^{n}\right)\right.
\end{aligned}
$$

by the Sobolev inequality applied to $\left\|e^{n}\right\|_{\infty}$.

Lastly, for the term $I_{1}$ we note from (31) that

$$
\delta^{2} U_{k}^{n}-\delta^{2} U_{k}^{n-1}=\frac{1}{h}\left(\eta_{k}^{n+1}-\eta_{k}^{n}-\left(\eta_{k}^{n}-\eta_{k}^{n-1}\right)\right)=\frac{\eta_{k}^{n+1}-2 \eta_{k}^{n}+\eta_{k}^{n-1}}{h},
$$

and hence

$$
I_{1}=\frac{1}{2 h} \sum_{k}\left(U_{k}^{n}+U_{k}^{n-1}\right) \delta^{2}\left(U_{k}^{n}-U_{k}^{n-1}\right) .
$$

Summing by parts we get

$$
\begin{aligned}
& I_{1}=-\frac{h^{-2}}{2 h} \sum_{k} {\left[U_{k+1}^{n}+U_{k+1}^{n-1}-U_{k}^{n}-U_{k}^{n-1}\right] } \\
& \cdot\left[U_{k+1}^{n}-U_{k+1}^{n-1}-\left(U_{k}^{n}-U_{k}^{n-1}\right)\right] .
\end{aligned}
$$

This can be rewritten as

$$
I_{1}=-\frac{1}{2 h} \sum_{k}\left[\left(\delta U_{k}^{n}\right)^{2}-\left(\delta U_{k}^{n-1}\right)^{2}\right]=-\frac{1}{2 h^{2}}\left[\left\|\delta U^{n}\right\|_{2}^{2}-\left\|\delta U^{n-1}\right\|_{2}^{2}\right] .
$$

Returning now to (50), we multiply it by $h^{2}$ to get

$$
\begin{aligned}
-\frac{1}{2}\left\|\delta U^{n}\right\|_{2}^{2}+\frac{1}{2}\left\|\delta U^{n-1}\right\|_{2}^{2} & \\
& -\frac{1}{4}\left(\left\|\eta^{n+1}\right\|_{2}^{2}+\left\|\eta^{n}\right\|_{2}^{2}\right)+\frac{1}{4}\left(\left\|\eta^{n}\right\|_{2}^{2}+\left\|\eta^{n-1}\right\|_{2}^{2}\right) \\
= & O\left(h\left(\mathscr{E}^{n}+\mathscr{E}^{n-1}\right)+h^{5}\right) .
\end{aligned}
$$


This completes the proof.

Proof of Theorem 2. Let us define $h=\Delta t=\Delta x$ and

$$
H^{n-1}=\frac{1}{2}\left\|\delta e^{n}\right\|_{2}^{2}+\frac{1}{2}\left\|\delta U^{n-1}\right\|_{2}^{2}+\frac{1}{4}\left(\left\|\eta^{n}\right\|_{2}^{2}+\left\|\eta^{n-1}\right\|_{2}^{2}\right) .
$$

Recall the definitions of the terms $\mathrm{II}^{n}$, $\mathrm{III}^{n}$ from Lemma 9. Adding the conclusions of Lemmas 9 and 10, we get

$$
\begin{aligned}
H^{n}+h\left(\mathrm{II}^{n}+\mathrm{III}^{n}\right)= & H^{n-1}+h\left(\mathrm{II}^{n-1}+\mathrm{III}^{n-1}\right) \\
& +O\left(h\left(\mathscr{E}^{n}+\mathscr{E}^{n-1}\right)+h^{3}\right),
\end{aligned}
$$

where, from (33),

$$
\mathscr{E}^{n}=\frac{1}{2}\left\|e^{n+1}\right\|_{2}^{2}+H^{n} .
$$

Now, for a (large) positive constant $\gamma$ (to be chosen below) set

$$
\hat{\mathscr{E}}^{n} \equiv \gamma\left\|e^{n+1}\right\|_{2}^{2}+H^{n}+h\left(\mathrm{II}^{n}+\mathrm{III}^{n}\right) .
$$

From (59) and Lemma 6 it follows that

$$
\begin{aligned}
\hat{\mathscr{E}}^{n} \leq & \gamma(1+c h)\left\|e^{n}\right\|_{2}^{2}+\gamma c_{T} h^{5}+c \gamma h\left(\left\|\eta^{n+1}\right\|_{2}^{2}+\left\|\eta^{n}\right\|_{2}^{2}\right) \\
& +H^{n-1}+h\left(\mathrm{II}^{n-1}+\mathrm{III}^{n-1}\right)+O\left(h\left(\mathscr{E}^{n}+\mathscr{E}^{n-1}\right)+h^{3}\right) .
\end{aligned}
$$

Now we estimate II $^{n}$, III $^{n}$ easily by

$$
\begin{aligned}
h\left|\mathrm{II}^{n}\right| & =\left|\frac{h}{2} \operatorname{Re} \sum_{k}\left(E\left(x_{k}, t^{n}\right)+E\left(x_{k}, t^{n+1}\right)\right)\left(\eta_{k}^{n+1}+\eta_{k}^{n}\right) \bar{e}_{k}^{n+1}\right| \\
& \leq c\left(\left\|E\left(t^{n}\right)\right\|_{\infty}+\left\|E\left(t^{n+1}\right)\right\|_{\infty}\right)\left\|\eta^{n+1}+\eta^{n}\right\|_{2}\left\|e^{n+1}\right\|_{2} \\
& \leq \frac{1}{16}\left(\left\|\eta^{n+1}\right\|_{2}^{2}+\left\|\eta^{n}\right\|_{2}^{2}\right)+c\left\|e^{n+1}\right\|_{2}^{2}
\end{aligned}
$$

(with a constant $c$ depending only on the data), and

$$
\begin{aligned}
h\left|\mathrm{III}^{n}\right| & \leq\left.\left|\frac{h}{2} \sum_{k}\left(N_{k}^{n+1}+N_{k}^{n}\right)\right| e_{k}^{n+1}\right|^{2} \mid \\
& \leq c\left\|e^{n+1}\right\|_{\infty}\left\|N^{n+1}+N^{n}\right\|_{2}\left\|e^{n+1}\right\|_{2} \\
& \leq c\left(\left\|N^{n+1}\right\|_{2}+\left\|N^{n}\right\|_{2}\right)\left\|e^{n+1}\right\|_{2}^{3 / 2}\left\|\delta e^{n+1}\right\|_{2}^{1 / 2}
\end{aligned}
$$

by the Sobolev inequality. Since the first factor is bounded by Lemma 3, we obtain

$$
h\left|\mathrm{III}{ }^{n}\right| \leq \frac{1}{8}\left\|\delta e^{n+1}\right\|_{2}^{2}+c\left\|e^{n+1}\right\|_{2}^{2}
$$

with $c$ depending only on the data. Adding (63) to (64), we obtain

$$
\begin{aligned}
h\left(\left|\mathrm{II}^{n}\right|+\left|\mathrm{III}^{n}\right|\right) & \leq \frac{1}{8}\left\|\delta e^{n+1}\right\|_{2}^{2}+\frac{1}{16}\left(\left\|\eta^{n+1}\right\|_{2}^{2}+\left\|\eta^{n}\right\|_{2}^{2}\right)+c\left\|e^{n+1}\right\|_{2}^{2} \\
& \leq \frac{1}{4} H^{n}+c\left\|e^{n+1}\right\|_{2}^{2}
\end{aligned}
$$

by the definition (58) of $H^{n}$. It follows that $\hat{\mathscr{E}}^{n}$ is strictly positive for a sufficiently large choice of $\gamma$, depending only on the data.

In fact, we can choose $\gamma$ large enough so that $\gamma>1$ and

$$
\widehat{\mathscr{E}}^{n} \geq \frac{c}{2}\left\|e^{n+1}\right\|_{2}^{2}+\frac{3}{4} H^{n}
$$


with a constant $c>0$ depending only on the data and on $\gamma$.

Hence, from (62),

$$
\hat{\mathscr{E}}^{n} \leq \hat{\mathscr{E}}^{n-1}+c_{T} \gamma h\left(\mathscr{E}^{n}+\mathscr{E}^{n-1}\right)+c_{T} \gamma h^{3} .
$$

Now from its definition, we have, since $\gamma>1$,

$$
\begin{aligned}
\mathscr{E}^{n} & =\frac{1}{2}\left\|e^{n+1}\right\|_{2}^{2}+H^{n}<\gamma\left\|e^{n+1}\right\|_{2}^{2}+H^{n} \\
& =\hat{\mathscr{E}}^{n}-h\left(\mathrm{II}^{n}+\mathrm{III}^{n}\right) \leq \hat{\mathscr{E}}^{n}+\frac{1}{4} H^{n}+c\left\|e^{n+1}\right\|_{2}^{2},
\end{aligned}
$$

where we have used (65). Since $H^{n} \leq \mathscr{E}^{n}$ by (60), we conclude that

$$
\frac{3}{4} \mathscr{E}^{n} \leq \hat{\mathscr{E}}^{n}+c\left\|e^{n+1}\right\|_{2}^{2} \leq c_{\gamma} \hat{\mathscr{E}}^{n}
$$

in view of (66). For any such (fixed) choice of $\gamma$, we obtain from (67)

$$
\left(1-c_{T} h\right) \hat{\mathscr{E}}^{n} \leq\left(1+c_{T} h\right) \hat{\mathscr{E}}^{n-1}+c_{T} h^{3} .
$$

It follows that for $h=\Delta t=\Delta x$ sufficiently small, depending only on $T$ and the data, we have

$$
\hat{\mathscr{E}}^{n} \leq c_{T}\left[\hat{\mathscr{E}}^{0}+h^{2}\right]
$$

Since $\left(\hat{\mathscr{E}}^{n}\right)^{1 / 2}$ is equivalent to $\left(\mathscr{E}^{n}\right)^{1 / 2}$, the first part of the proof is complete.

It remains to estimate $\mathscr{E}^{0}$. From (29), (30) and (9), (10) we have

$$
e_{k}^{0}=0, \quad \eta_{k}^{0}=0, \quad \eta_{k}^{1}=O\left(h^{2}\right)
$$

Thus, $\left\|\eta^{1}\right\|_{2}^{2}+\left\|\eta^{0}\right\|_{2}^{2}=O\left(h^{4}\right)$. From Lemma 6 with $n=0,\left\|e^{1}\right\|_{2}^{2}=O\left(h^{5}\right)$, and hence

$$
\left\|\delta e^{1}\right\|_{2}^{2}=h^{-1} \sum_{k=1}^{J}\left|e_{k+1}^{1}-e_{k}^{1}\right|^{2} \leq 4 h^{-1} \sum_{k=1}^{J}\left|e_{k}^{1}\right|^{2}=O\left(h^{3}\right) .
$$

Finally, we bound $\left\|\delta U_{k}^{n}\right\|_{2}$. We multiply the definition of $U_{k}^{n}$ by $U_{k}^{n}$, sum over $k$, and then sum by parts to get

$$
\left\|\delta U^{0}\right\|_{2}^{2}=-\sum_{k=1}^{J} U_{k}^{0}\left(\eta_{k}^{1}-\eta_{k}^{0}\right)=\sum_{k=1}^{J-1} \sum_{j=1}^{J-1} G\left(x_{k}, x_{j}\right) \eta_{k}^{1} \eta_{j}^{1},
$$

where we have used Lemma 4 again. Since $G$ is continuous, it follows from general considerations (or from explicit computation, using $\eta_{k}^{1}=O\left(h^{2}\right)$ ) that the last expression is $O\left(h^{2}\right)$, and this completes the proof.

\section{BIBLIOGRAPHY}

1. H. Added and S. Added, Equations of Langmuir turbulence and nonlinear Schrödinger equation: Smoothness and approximation, J. Funct. Anal. 79 (1988), 183-210.

2. M. Delfour, M. Fortin, and G. Payne, Finite difference solution of a nonlinear Schrödinger equation, J. Comput. Phys. 44 (1981), 277-288.

3. J. Gibbons, S. G. Thornhill, M. J. Wardrop, and D. Ter Harr, On the theory of Langmuir solitons, J. Plasma Phys. 17 (1977), 153-170.

4. R. Glassey and J. Schaeffer, Convergence of a second-order scheme for semilinear hyperbolic equations in $2+1$ dimensions, Math. Comp. 56 (1991), 87-106.

5. G. L. Payne, D. R. Nicholson, and R. M. Downie, Numerical solution of the Zakharov equations, J. Comput. Phys. 50 (1983), 482-498. 
6. J. M. Sanz-Serna, Methods for the numerical solution of the nonlinear Schrödinger equation, Math. Comp. 43 (1984), 21-27.

7. S. Schochet and M. Weinstein, The nonlinear Schrödinger limit of the Zakharov equations governing Langmuir turbulence, Comm. Math. Phys. 106 (1986), 569-580.

8. W. Strauss and L. Vazquez, Numerical solution of a nonlinear Klein-Gordon equation, J. Comput. Phys. 28 (1978), 271-278.

9. C. Sulem and P. L. Sulem, Regularity properties for the equations of Langmuir turbulence, C. R. Acad. Sci. Paris Sér. A Math. 289 (1979), 173-176.

10. C. Sulem, P. L. Sulem, and H. Frisch, Tracing complex singularities with spectral methods, J. Comput. Phys. 50 (1983), 138-161.

11. V. E. Zakharov, Collapse of Langmuir waves, Soviet Phys. JETP 35 (1972), 908-912.

Department of Mathematics, Indiana University, Bloomington, Indiana 47405

E-mail address: glassey@ucs.indiana.edu 